\title{
PIAS1 interacts with the KRAB zinc finger protein, ZNF133, via zinc finger motifs and regulates its transcriptional activity
}

\author{
Sang-Jin Lee ${ }^{1 *}$, Jae-Rin Lee ${ }^{1 *}$, \\ Hwa-Sun Hahn', Young-Hoon Kim ${ }^{1}$, \\ Jin-Hyun Ahn ${ }^{1}$, Chang-Dae Bae ${ }^{1}$, \\ Jun-Mo Yang ${ }^{2}$ and Myong-Joon Hahn ${ }^{1,3}$ \\ ${ }^{1}$ Department of Molecular Cell Biology \\ Center for Molecular Medicine \\ Samsung Biomedical Research Institute \\ Sungkyunkwan University School of Medicine \\ Suwon 440-746, Korea \\ ${ }^{2}$ Department of Dermatology \\ Sungkyunkwan University School of Medicine \\ Samsung Medical Center \\ Seoul 135-710, Korea \\ ${ }^{3}$ Corresponding author: Tel, 82-31-299-6221; \\ Fax, 82-31-299-6239; E-mail, mjhahn@yurim.skku.ac.kr \\ *These authors contributed equally to this work.
}

Accepted 7 May 2007

Abbreviations: HDAC, histone deacetylases; KRAB, Krüppelassociated box; PIAS1, protein inhibitor of activated STAT1; TIF1 $\beta$, transcriptional intermediary factor $1 \beta$; TSA, trichostatin $A$; ZNF133, zinc finger protein 133

\begin{abstract}
Zinc finger protein 133 (ZNF133) is composed of a Krüppel-associated box (KRAB) domain and 14 contiguous zinc finger motifs. ZNF133 is regarded as a transcriptional repressor because the KRAB domain has potent repressor activity and the zinc finger motifs usually act in binding to DNA. However, we found that the zinc finger motifs of ZNF133 also possessed transcriptional repressor activity. By two-hybrid screening assay, we found that the zinc finger motifs of ZNF133 interacted with protein inhibitor of activated STAT1 (PIAS1). PIAS1 enhanced the transcriptional repression activity of ZNF133 through the zinc finger motifs. This effect of PIAS1 was relieved by an inhibitor of the histone deacetylases (HDACs). These results demonstrate that the transcriptional repressor activity of ZNF133 is regulated by both the KRAB domain and the zinc finger motifs, and that the repressive effect by zinc finger motifs is mediated by PIAS1.
\end{abstract}

Keywords: protein inhibitors of activated STAT; re- pressor proteins; transcription, genetic; zinc fingers; ZNF133 protein

\section{Introduction}

The human genome encodes approximately 290 Krüppel-associated box (KRAB) zinc finger proteins, but little is known about the biological functions of these proteins. Proteins in this family are characterized by the presence of the KRAB domain near the amino termini and multiple zinc finger motifs (4 to 30 ) at their carboxy termini. The $\mathrm{KRAB}$ domain is a potent repressor domain that spans approximately 75 amino acids. Repression by KRAB domains is mediated by the binding of the transcriptional co-repressor, transcriptional intermediary factor $1 \beta$ (TIF1 $\beta$ ) which is also known as KRAB-associated protein (KAP-1) or KRAB-A interacting proteins 1 (KRIP-1) (Friedman et al., 1996; Kim et al., 1996; Moosmann et al., 1996). As this co-repressor protein interacts with KRAB domains from multiple origins, it is regarded as the common co-repressor of the KRAB-zinc finger proteins. The KRAB-TIF1 $\beta$ complex recruits heterochromatin-associated proteins such as HP1 (Ryan et al., 1999) and SETDB1 (Schultz et al., 2002), which methylates lysine residue (K9) on the tail of histone $3(\mathrm{H} 3)$. TIF1 $\beta$ also interacts with histone deacetylase complexes- NcoR1 (Underhill et al., 2000) and NuRD (Schultz et al., 2001). By associating with those proteins through the KRAB domain, KRAB zinc finger proteins are thought to exert transcriptional repressor activity on their targets, which are recognized by zinc finger motifs. Most zinc finger motifs are involved in DNA binding; however, some of the KRAB-zinc finger family proteins, including ZBRK1, have been shown to bind both DNA and protein via its zinc finger motifs (Zheng et al., 2000).

Zinc finger protein 133 (ZNF133) was identified as one of the KRAB-zinc finger family proteins (Tommerup and Vissing, 1995). Like most proteins of this family, the biological function, mechanism of regulation, and target sequence of this protein largely remain unknown. The KRAB domain of ZNF133 was reported to have transcriptional repression activity (Vissing et al., 1995) and to interact with TIF1 $\beta$ (Friedman et al., 1996). In several microarray studies, the expressions of 
ZNF133 were reported to be elevated in chronic myeloid leukemia (Li et al., 2002) and neuroblastoma cells (Heiskanen et al., 2000), as well as by INF $\gamma$ stimulation (Andersen et al., 2003); these findings suggest its possible role in oncogenesis and/or immune response. In this study, we show that, in addition to the KRAB domain, the zinc finger motifs of ZNF133 also mediate transcriptional repression via interaction with protein inhibitor of activated STAT1 (PIAS1), and this effect requires histone deacetylase (HDAC) activity.

\section{Materials and Methods}

\section{Plasmids}

Full-length cDNAs encoding ZNF133, TIF1 $\beta$, and PIAS1 were cloned from human thymus cDNA (Clontech) into pCMV6-HA, myc, and SRT (Lee et al., 2001), respectively. cDNAs encoding partial fragments of ZNF133 (amino acids 1-213 and 214-654) were subcloned into pCMV6-HA, and the C-terminal region of PIAS1 (424-651) was subcloned into pCMV6-SRT. Mammalian expression plasmids for GAL4-DNA-binding (DB) domain fusions GAL4-ZNF133 (1-654), GAL4-ZNF133 (1-213), and GAL4-ZNF133 (214-654) were generated on a pSV2-GAL4 background ( $\mathrm{pGH} 250$ ) (Kim et al., 2005). For luciferase assay, (GAL4) 5 / TK-Luc reporter plasmid (Rountree et al., 2000) was used.

\section{Yeast two-hybrid screening}

The bait plasmid was generated by subcloning a cDNA fragment encoding the zinc finger motifs (214-654) into the pLexA vector (Clontech). The EGY48 yeast strain was transformed by the bait plasmid and two-hybrid cDNA library DNA derived from human fetal brain (Clontech). About $10^{6}$ transformants were screened and positive clones were selected for growth on plates lacking tryptophan, histidine, uracil, and leucine, and were subsequently tested for $\beta$-galactosidase activity.

\section{Cell culture}

HEK293 and HeLa cells were grown in DMEM supplemented with $10 \%$ FBS. For immunoprecipitation, HEK293 cells were transfected with the expression plasmids by the calcium phosphate precipitation method. HEK293 and HeLa cells were used for the luciferase assay and indirect immnofluorescence assay (IFA), respectively, and were transfected with LipofectAMINE reagents (Invitrogen).

\section{Immunoprecipitation and immunoblotting}

HEK293 cells were cotransfected with the HAtagged plasmids encoding either full-length, 1-213, or 214-654 amino acids of ZNF133 and the SRT-tagged plasmids encoding full-length or 424-651 amino acids of PIAS1. Forty-eight hours after transfection, cells were lysed with a lysis buffer (50 mM Tris- $\mathrm{HCl}, \mathrm{pH} 8.0,0.1 \%$ Triton X-100, $50 \mathrm{mM}$ sodium fluoride, $5 \mathrm{mM}$ sodium pyrophosphate, $1 \mathrm{mM}$ PMSF, $1 \mathrm{mM}$ sodium orthovanadate, $2 \mathrm{mM}$ leupeptin). After clearing by centrifugation, total cell lysates were incubated with anti-SRT antibody. After $1 \mathrm{~h}$ incubation at $4^{\circ} \mathrm{C}$, protein A-Sepharose beads were added and adsorbed for another hour. Immune complexes were pelleted, washed twice with lysis buffer, and then washed twice with lysis buffer containing 100 $\mathrm{mM} \mathrm{NaCl}$. Precipitated proteins were resolved by SDS-PAGE and probed with the appropriate antibodies.

\section{Immnofluorescence assay (IFA)}

HeLa cells were grown on microscope slide cover slips and transfected with plasmids encoding SRTtagged ZNF133 and HA-tagged PIAS1. Forty-eight hours after transfection, cells were fixed in PBS with $1 \%$ paraformaldehyde and permeabilized with $0.1 \%$ Triton $\mathrm{X}-100$ in PBS. After three washes in PBS, cells were incubated with anti-SRT mouse monoclonal antibody and biotin-conjugated anti-HA rat monoclonal antibody, followed by incubation with rhodamine-conjugated goat anti-mouse $\lg G$ and FITC-conjugated streptavidin.

\section{Results}

\section{Transcriptional repressor activity by the zinc finger motifs of ZNF133}

To assess whether the zinc finger motifs of ZNF133 contribute to transcriptional repressor activity, we performed the GAL4 DNA-tethering assays (Oh et al., 2005; Kim et al., 2006). For this experiment, GAL4 DNA-binding domain fusion constructs containing zinc finger motifs (214-654), the N-terminal region (1-213), and full-length ZNF133 were generated (Figure 1A). Immunoblot analysis with cell extracts prepared from transfected HEK293 cells confirmed that the fusion proteins were expressed at similar levels (Figure 1B). Next, HEK293 cells were cotransfected with a luciferase reporter gene containing the GAL4 binding sites and the GAL4-ZNF133 fusions, and the luciferase assays were subsequently per- 
A

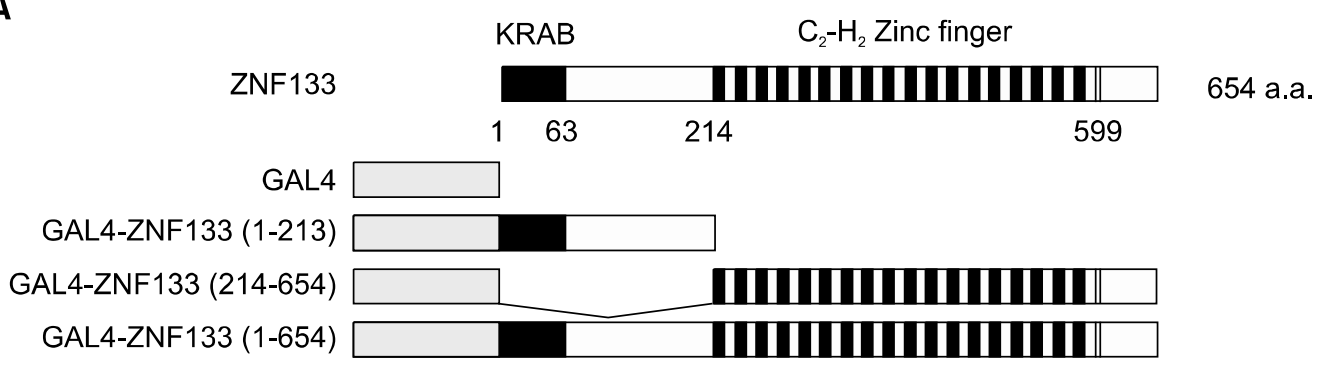

B

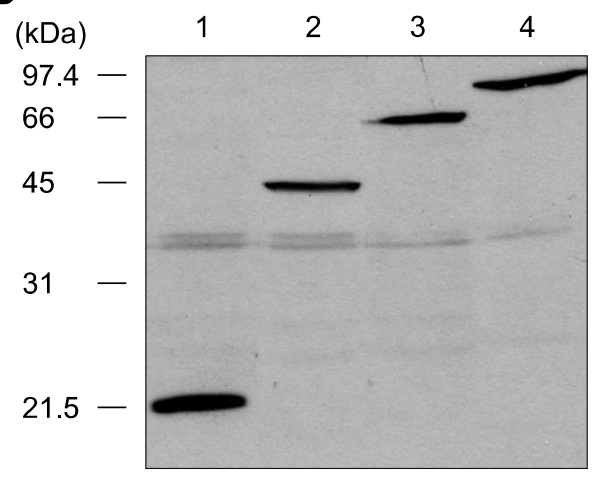

C

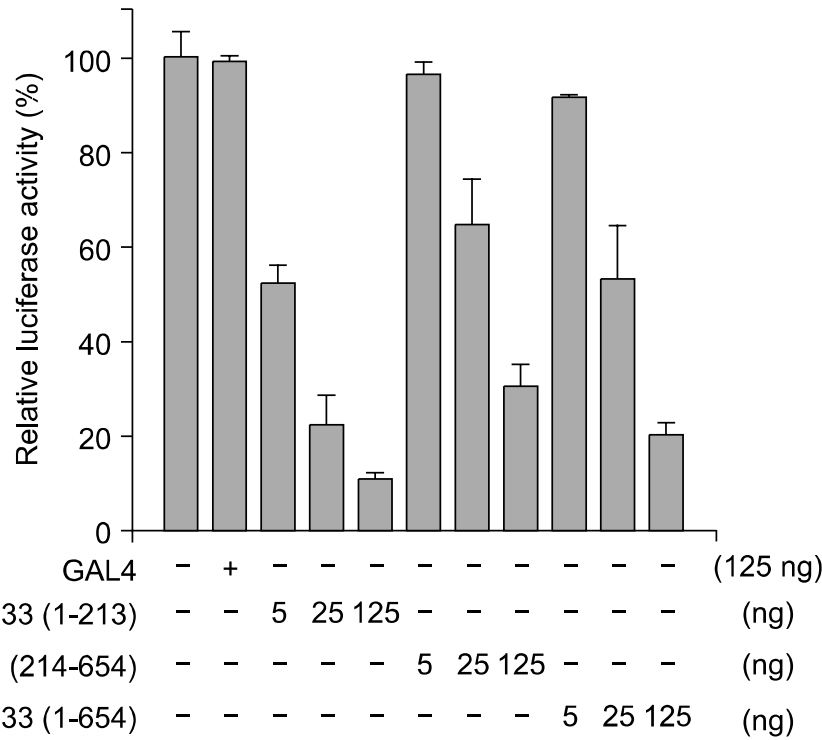

Figure 1. The zinc finger motifs of ZNF133 have transcriptional repressor activity. (A) The domain structure of ZNF133. The N-terminal KRAB domain (1-63) and 14 zinc finger motifs (214-599) at the C-terminal half region are indicated. GAL4-DNA-binding (DB) alone and GAL4-ZNF133 fusion constructs used in this study are shown. (B) Expression levels of GAL4-ZNF133 fusion proteins. The protein bands of GAL4-DB domain alone (lane 1), GAL4-ZNF133 (1-213) (lane 2), GAL4-ZNF133 (214-654) (lane 3), or GAL4-ZNF133 (1-654) (lane 4) were detected with anti-GAL4 antibody. Protein size markers are indicated on the left side. (C) Transcriptional repression activity of ZNF133. HEK293 cells were cotransfected with $50 \mathrm{ng}$ of luciferase reporter plasmid containing a (GAL4) $5 /$ TK-Luc gene and $125 \mathrm{ng}$ of plasmid encoding GAL4-DB alone, with increasing amounts $(5,25,125 \mathrm{ng})$ of plasmid encoding the indicated GAL4-ZNF133 fusion proteins. At $24 \mathrm{~h}$ after transfection, total cell extracts were prepared and assayed for luciferase activity. pCMV- $\beta$-gal plasmid was used as an internal transfection control. Transfection efficiency was normalized by $\beta$-galactosidase activity. The results, expressed as luciferase activities \pm standard errors, are representative of at least three experiments.

formed. The results showed that GAL4-ZNF133 (1-213), GAL4-ZNF133 (214-654), and GAL4ZNF133 (1-654) each efficiently repressed transcription of the reporter gene in dose-dependent manners. These data demonstrate that, like the $K R A B$ domain, the zinc finger motifs also have the potential to repress transcription and may contribute to the overall transcriptional repressor activity of ZNF133.

\section{PIAS1 interacts with the zinc finger motifs of ZNF133}

In an attempt to investigate the mechanism of ZNF133 zinc finger motif-mediated transcriptional repression, a yeast two-hybrid cDNA library derived from human fetal brain was screened with the zinc finger motifs of ZNF133 as bait. Sequence analysis revealed that one of the positive clones contained the C-terminal region (424-651) of PIAS1 (Figure 2A). We further investigated the interaction of ZNF133 with PIAS1 in mammalian cells using coimmunoprecipitation assays. First, ZNF133 was coexpressed with either full-length PIAS1 or the C-terminal region (424-651) of PIAS1 in HEK293 cells. Immunoprecipitation of full-length PIAS1 or PIAS1 (424-651) resulted in coprecipitation of ZNF133, demonstrating that ZNF133 binds to the $C$-terminal region of PIAS1 (Figure 2B). A reciprocal experiment was also conducted by using expression plasmids for the KRAB domain or the zinc finger motifs of ZNF133, in combination with PIAS1 (424-651). The results 
A

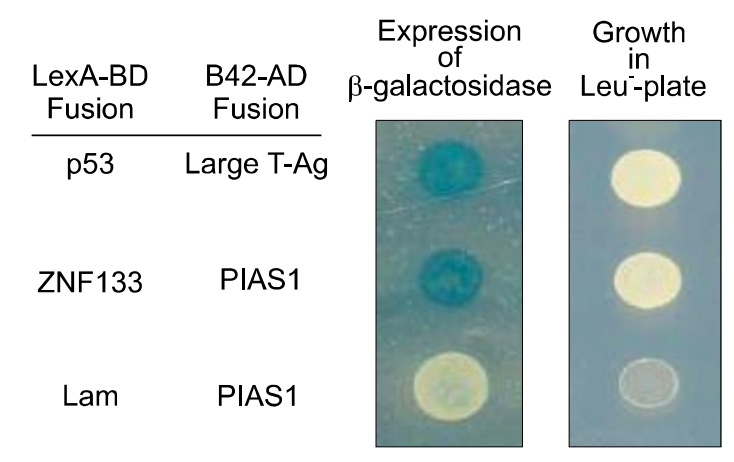

B

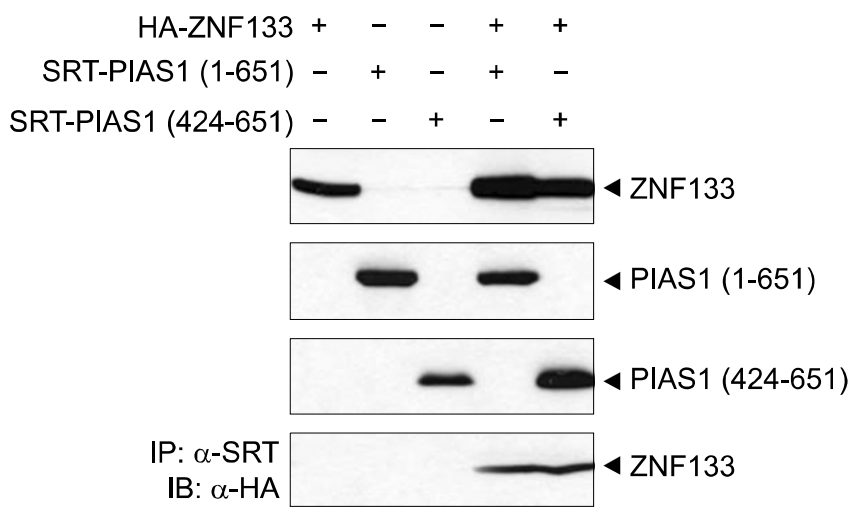

C

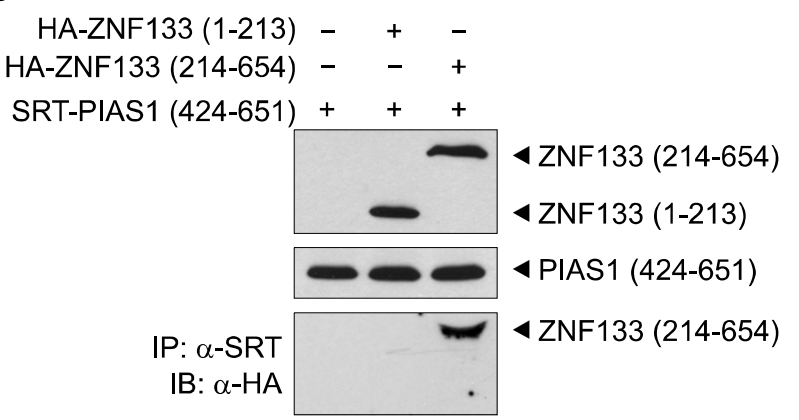

\section{D}
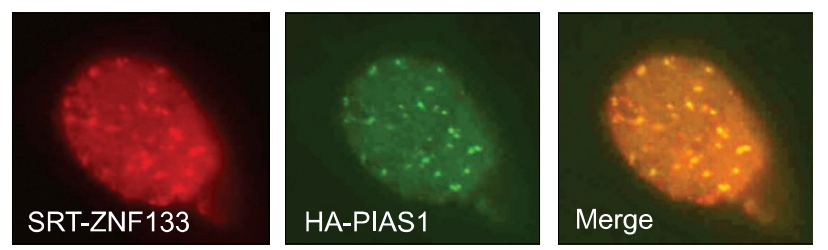

Figure 2. PIAS1 is a novel binding partner of ZNF133. (A) Interaction of ZNF133 and PIAS1 in a yeast two-hybrid system. The human fetal brain cDNA library was screened using ZNF133 (214-654) as bait. The binding between ZNF133 and PIAS1 was confirmed using the lacZ or Leu reporter system. pLexA-DNA binding domain (BD)-p53 and pB42 activation domain (AD)-T plasmids were used as positive control, and pLexA-Lam plasmid was used as a negative control for their interaction. (B) ZNF133 interacts with the C-terminal region of PIAS1 in transfected cells. HEK293 cells were transiently transfected with HA-tagged ZNF133 together with full-length SRT-tagged PIAS1 (1-651) or the C-terminal region (424-651) of SRT-tagged PIAS1 as indicated. For immunoblot analysis of total cell lysates, $100 \mu \mathrm{g}$ of cell lysates was resolved in SDS-PAGE gel and probed with anti-HA or anti-SRT antibody, respectively. For immunoprecipitation, total cell lysates were incubated with anti-SRT antibody and pelleted with protein A beads. Immunoprecipitants were analyzed using anti-HA antibody. (C) The zinc finger motifs of ZNF133 are required for interaction with PIAS1 in transfected cells. HEK293 cells were transiently transfected with SRT-PIAS1 (424-651) together with HA-ZNF133 (1-213) or the zinc finger domain of HA-tagged ZNF133 (214-654). Immunoblot analysis and immunoprecipitation processes were performed as described in A. (D) Colocalization of ZNF133 and PIAS1. Transiently expressed HA-PIAS1 (green) and SRT-ZNF133 (red) were colocalized at nuclear speckle structures in HeLa cells.

showed that immunoprecipitation of PIAS1 (424$651)$ resulted in coprecipitation of the zinc finger motifs (214-654) of ZNF133, but not the KRAB domain (1-213) of ZNF133 (Figure 2C). Consistent with the finding from the yeast two-hybrid interaction assays, these results demonstrate that the zinc finger motifs of ZNF133 specifically bind to the C-terminal region of PIAS1. Intracellular localizations of these two proteins were also tested by IFA. SRT-ZNF133 and HA-PIAS1 were coexpressed in HeLa cells, and double-label IFA was performed with antibodies for SRT and HA tags. The results showed that both proteins were completely colocalized as nuclear speckles (Figure 2D), which supports our finding that PIAS1 interacts with ZNF133 in the nucleus.

\section{PIAS1 enhances transcriptional repression by the zinc finger motifs of ZNF133}

To determine the functional role of PIAS1 in transcriptional regulation by ZNF133, we investigated the effect of PIAS1 on ZNF133-mediated transcriptional repression. For this, we performed GAL4 DNA-tethering assays similar to those described in Figure 1C. We found that expression of PIAS1 augmented GAL4-ZNF133-mediated transcriptional repression in a dose-dependent manner, whereas it had little effect on the activity of GAL4 alone (Figure 3A). Similar effects of PIAS1 were observed for GAL4-ZNF133 (214-654)-mediated transcriptional repression (Figure $3 \mathrm{~B}$ ). Expression of TIF1 $\beta$, which targets the KRAB domain and acts as a co-repressor, had no effect on the ZNF133 (214-654)-mediated transcriptional repression (Figure $3 \mathrm{~B})$. These results demonstrate that PIAS1 potentiates ZNF133-induced transcriptional repres- 
A

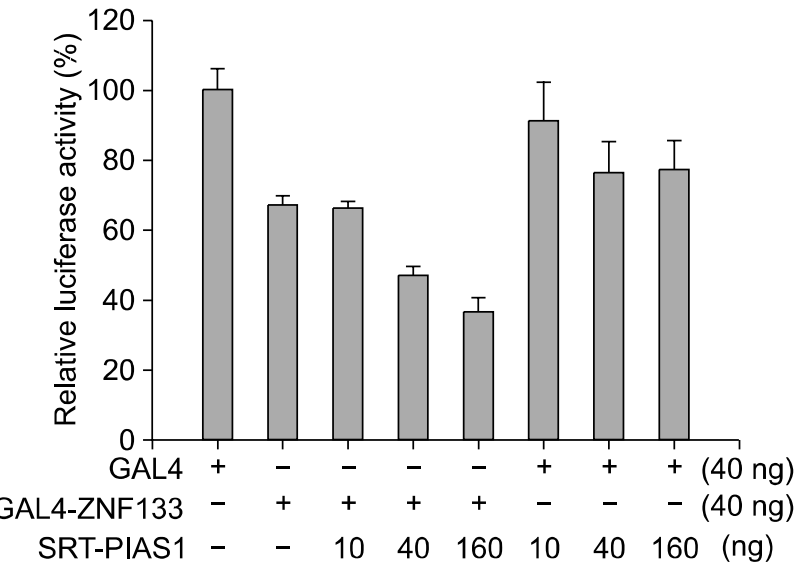

B

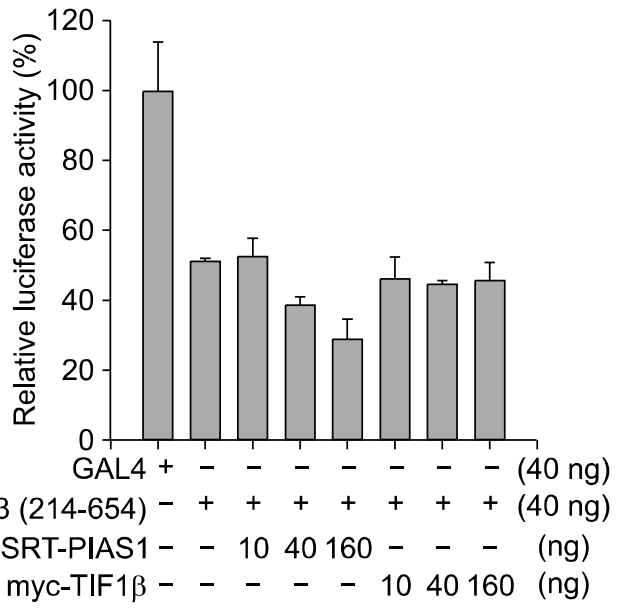

Figure 3. PIAS1 enhances the transcriptional repression activity of ZNF133. (A) HEK293 cells were transiently transfected with increasing amount of SRT-PIAS1 (10 to $160 \mathrm{ng}$ ) with $40 \mathrm{ng}$ of plasmid encoding GAL4-DB alone or GAL4-ZNF133 (1-654). After $24 \mathrm{~h}$ of transfection, luciferase activity was performed as in Figure $1 \mathrm{C}$. Mean values and standard errors of luciferase activity from at least three independent experiments are shown. (B) Increasing amounts of plasmid encoding PIAS1 (10 to $160 \mathrm{ng}$ ) or TIF1 $\beta$ (10 to $160 \mathrm{ng}$ ) together with GAL4-ZNF133 (214-654) were transfected into HEK293 cells. After $24 \mathrm{~h}$, luciferase activity was measured as described in $\mathrm{A}$.

A

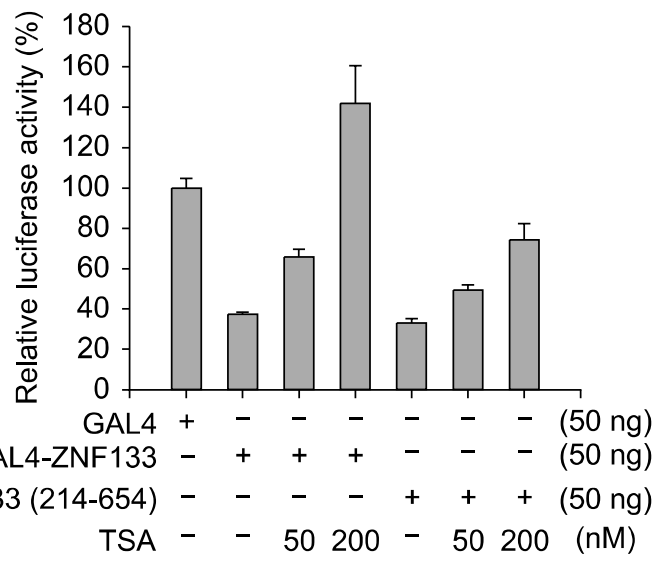

B

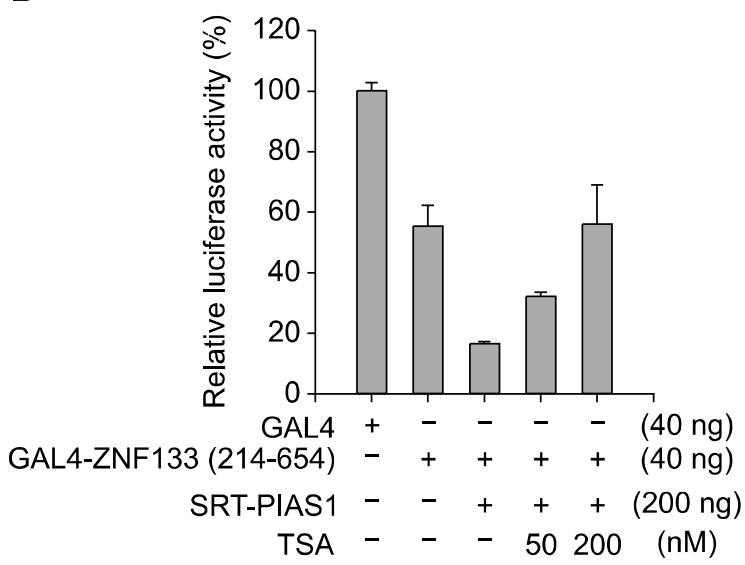

Figure 4. The effect of PIAS1 on the transcriptional repression activity of ZNF133 is mediated through histone deacetylase. (A) HEK293 cells were transfected with $50 \mathrm{ng}$ of GAL4-DB alone, GAL4-ZNF133 (1-654), or GAL4-ZNF133 (214-654). After $24 \mathrm{~h}$ of transfection, cells were incubated with 50 or 200 $\mathrm{nM}$ of TSA for $3 \mathrm{~h}$, after which luciferase activity was measured. The values were normalized according to $\beta$-galactosidase activity. The mean values from at least three independent experiments are shown. (B) $40 \mathrm{ng}$ of plasmid encoding GAL4-ZNF133 (214-654) was transfected in HEK293 cells with $200 \mathrm{ng}$ of plasmid encoding PIAS1. After $24 \mathrm{~h}$, TSA (50 or $200 \mathrm{nM}$ ) was treated for $3 \mathrm{~h}$. The values of luciferase activity were obtained as described in A.

sion by binding to the zinc finger motifs.

\section{PIAS1-mediated augmentation of transcriptional repression by ZNF133 requires histone deacetylase activity}

PIAS1 has been shown to interact with various transcription factors and regulate their transcriptional activities through several mechanisms, including recruitment of other transcriptional coregulators (Shuai and Liu, 2005). To investigate whether histone deacetylase, which interacts with PIAS proteins (Tussie-Luna et al., 2002; Long et al., 2003; Gross et al., 2004), mediates the regulatory activity of PIAS1 on ZNF133, we investigated the effect of the HDAC inhibitor, trichostatin A (TSA), on this process. The results of GAL4 DNA tethering assays showed that TSA treatment completely reversed the repression activities of both full-length ZNF133 and ZNF133 (214-654) (Figure 4). Furthermore, TSA treatment also completely inhibited the augmentation effect of PIAS1 


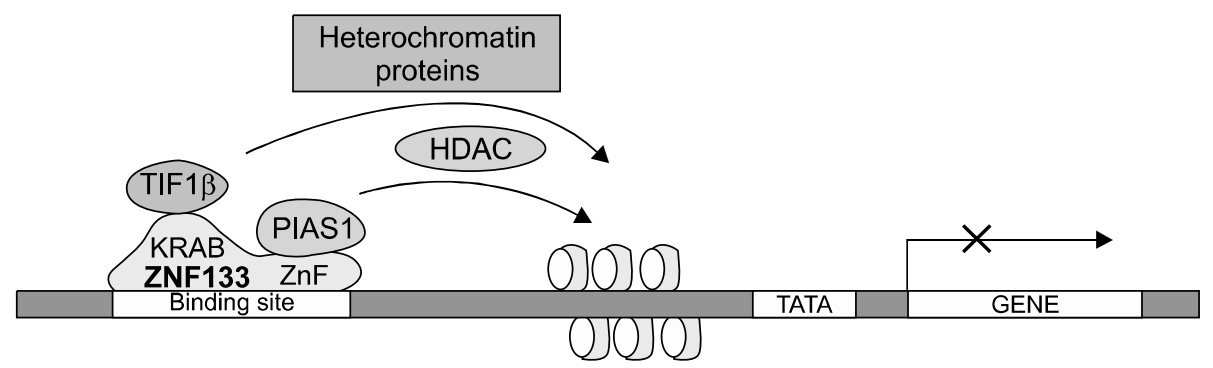

Figure 5. Proposed mechanism for the transcriptional modulation of ZNF133 through PIAS1. ZNF133 may bind to specific DNA sequences through its zinc finger motifs. After binding, TIF1 $\beta$ can associate with KRAB domain of ZNF133 and suppress target gene transcription. In our model, zinc finger motifs (ZnF) of ZNF133 appear to have another role in transcriptional repression besides DNA binding activity. ZNF133 interacts with PIAS1 via zinc finger motifs and may inhibit transcription of target genes by forming complex with other co-repressors such as HDAC through PIAS1.

in ZNF133 (214-654)-mediated transcriptional repression (Figure 4B). These data suggest that recruitment of HDACs via PIAS1 may play a role in transcriptional repression by the zinc finger motifs of ZNF133.

\section{Discussion}

The KRAB zinc finger proteins make up one of the largest families of transcription factors. KRAB domains of this family of proteins have potent transcriptional repressor activity (Vissing et al., 1995). Because TIF1 $\beta$ interacts with many KRAB domains of this family, TIF1 $\beta$ is proposed to act as the common co-repressor of KRAB zinc finger proteins (Friedman et al., 1996; Kim et al., 1996; Moosmann et al., 1996).

Even though the role of the KRAB domain in transcriptional regulation has been characterized in depth, the role of zinc fingers has not yet been extensively studied. In general, this motif is required for DNA binding. However, in some proteins, this motif is also involved in binding with other proteins (Zheng et al., 2000). In this study, using the GAL4-fusion proteins and a reporter gene containing GAL4 binding sites upstream of the minimal TK promoter, we found that, in addition to the well-known KRAB domain, the zinc finger motifs of ZNF133 also have transcriptional repressor activity. Furthermore, using a two-hybrid screening assay with the zinc finger motifs as bait, we also found that a transcriptional regulator, PIAS1, interacts with the zinc finger motifs of ZNF133.

PIAS1 was originally identified as an inhibitor of STAT1 (Liu et al., 1998). A recent study showed that PIAS-deficient mice increase the expression of a subset of interferon-induced genes and increase the antiviral activity of interferon (Liu et al., 2004), thus supporting the physiological role of PIAS1 as a STAT1 regulator in the innate immune response. However, PIAS1 has also been reported to bind and, therefore, regulate numerous transcription factors, including NF-kB (Liu et al., 2005) and p53 (Kahyo et al., 2001; Megidish et al., 2002; Schmidt and Muller, 2002), through several mechanisms. DNA binding of STAT1 and NF-KB is inhibited by PIAS1. PIASy, a member of the PIAS family, represses the transcriptional activity of STAT1 by recruiting histone deacetylases (Liu et al., 2001).

Our data show that PIAS1 specifically binds to the zinc finger motifs of ZNF133, and this interaction augments ZNF133-mediated transcriptional repression. Furthermore, when we investigated whether HDAC inhibition could restore the PIAS1-induced repression, as seen with androgen receptor (Tan et al., 2000; Nishida and Yasuda, 2002), it was found that TSA treatment efficiently inhibited the PIAS1-mediated repressor activities of the zinc finger motifs in a dose-dependent manner. Our data suggest that PIAS1 binds the zinc finger motifs and represses the transcription from the unidentified target sequence(s) by recruiting HDACs (Figure 5).

Although the biological functions of ZNF133 and most of the KRAB zinc finger proteins remain elusive, it should also be noted that the expression of ZNF133 was reportedly increased by INF $\gamma$ (Andersen et al., 2003). Considering that PIAS1 regulates expression by interferons, it is tempting to speculate that PIAS1 recognizes its targets through transcription factors including ZNF133. Microarray studies have shown that expression of ZNF133 was elevated in chronic myeloid leukemia (Li et al., 2002) and neuroblastoma cells (Heiskanen et al., 2000). With the knowledge that PIAS1 regulates the transcriptional activity of p53 (Kahyo et al., 2001; Megidish et al., 2002; Schmidt and Muller, 2002), it is believed that ZNF133 could 
have roles in oncogenesis. Further studies are required to clarify those possibilities; in particular, identification of the target sequence would provide insight into the functional role of this protein.

\section{Acknowledments}

This work was supported by grants from the 2001 Good Health R \& D Project of the Ministry of Health (01-PJ3PG6-01GN12-0001) and from the Basic Research Program (R01-2004-0011007-0) of the Korea Science and Engineering Foundation (KOSEF) to M. J. H., and a grant from Ubiquitome Reaserch Program of KOSEF to J. H. A.

\section{References}

Andersen K, Smith-Sørensen B, Pedersen KB, Hovig E, Myklebost O, Fodstad O, Maelandsmo GM. Interferongamma suppresses $\mathrm{S} 100 \mathrm{~A} 4$ transcription independently of apoptosis or cell cycle arrest. Br J Cancer 2003;88: 1995-2001

Friedman JR, Fredericks WJ, Jensen DE, Speicher DW, Huang XP, Neilson EG, Rauscher FJ 3rd. KAP-1, a novel corepressor for the highly conserved KRAB repression domain. Genes Dev 1996;10:2067-78

Gross M, Yang R, Top I, Gasper C, Shuai K. PIASy-mediated repression of the androgen receptor is independent of sumoylation. Oncogene 2004;23:3059-66

Heiskanen MA, Bittner ML, Chen Y, Khan J, Adler KE, Trent JM, Meltzer PS. Detection of gene amplification by genomic hybridization to cDNA microarrays. Cancer Res 2000;60: 799-802

Kahyo T, Nishida T, Yasuda H. Involvement of PIAS1 in the sumoylation of tumor suppressor p53. Mol Cell 2001; 8:713-8

Kim JM, Kim SJ, Lee HC, Kim KS. Development of ligand-dependent regulatory system and its application to gene therapy of insulin-dependent diabetes mellitus. Exp Mol Med 2006;38:385-92

Kim SS, Chen YM, O'Leary E, Witzgall R, Vidal M, Bonventre JV. A novel member of the RING finger family, KRIP-1, associates with the KRAB-A transcriptional repressor domain of zinc finger proteins. Proc Natl Acad Sci USA 1996;93:15299-304

Kim YE, Kim DY, Lee JM, Kim ST, Han TH, Ahn JH. Requirement of the coiled-coil domain of PML-RAR $\alpha$ oncoprotein for localization, sumoylation, and inhibition of monocyte differentiation. Biochem Biophys Res Commun 2005;330:746-54

Lee JR, Chang YY, Hahn MJ. Development of a new epitope tag recognized by a monoclonal antibody to Rickettsia typh. BioTechniques 2001;31:541-5

Li H, Jie S, Zou P, Zoub G. cDNA microarray analysis of chronic myeloid leukemia. Int J Hematol 2002;75:388-93

Liu B, Liao J, Rao X, Kushner SA, Chung CD, Chang DD, Shuai K. Inhibition of Stat1-mediated gene activation by
PIAS1. Proc Natl Acad Sci USA 1998;95:10626-31

Liu B, Gross M, ten Hoeve J, Shuai K. A transcriptional corepressor of Stat1 with an essential LXXLL signature motif. Proc Natl Acad Sci USA 2001;98:3203-7

Liu B, Mink S, Wong KA, Stein N, Getman C, Dempsey PW, $\mathrm{Wu} \mathrm{H}$, Shuai K. PIAS1 selectively inhibits interferoninducible genes and is important in innate immunity. Nat Immunol 2004;5:891-8

Liu B, Yang R, Wong KA, Getman C, Stein N, Teitell MA, Cheng $\mathrm{G}$, Wu $\mathrm{H}$, Shuai K. Negative regulation of NF-кB signaling by PIAS1. Mol Cell Biol 2005;25:1113-23

Long J, Matsuura I, He D, Wang G, Shuai K, Liu F. Repression of Smad transcriptional activity by PIASy, an inhibitor of activated STAT. Proc Natl Acad Sci USA 2003;100:9791-6

Megidish T, Xu JH, Xu CW. Activation of p53 by protein inhibitor of activated Stat1 (PIAS1). J Biol Chem 2002; 277:8255-9

Moosmann P, Georgiev O, Le Douarin B, Bourquin JP, Schaffner W. Transcriptional repression by RING finger protein TIF1 beta that interacts with the KRAB repressor domain of KOX1. Nucleic Acids Res 1996;24:4859-67

Nishida T, Yasuda H. PIAS1 and PIASx $\alpha$ function as SUMO-E3 ligases toward androgen receptor, and repress androgen receptor-dependent transcription. J Biol Chem 2002;277:41311-7

Oh JH, Do HJ, Yang HM, Moon SY, Cha KY, Chung HM, Kim $\mathrm{JH}$. Identification of a putative transactivation domain in human Nanog. Exp Mol Med 2005;37:250-4

Rountree MR, Bachman KE, Baylin SB. DNMT1 binds HDAC2 and a new co-repressor, DMAP1, to form a complex at replication foci. Nat Genet 2000;25:269-77

Ryan RF, Schultz DC, Ayyanathan K, Singh PB, Friedman JR, Fredericks WJ, Rauscher FJ 3rd. KAP-1 corepressor protein interacts and colocalizes with heterochromatic and euchromatic HP1 proteins: a potential role for Kruppelassociated box-zinc finger proteins in heterochromatinmediated gene silencing. Mol Cell Biol 1999;19:4366-78

Schmidt D, Muller S. Members of the PIAS family act as SUMO ligases for c-Jun and p53 and repress p53 activity. Proc Natl Acad Sci USA 2002;99:2872-7

Schultz DC, Friedman JR, Rauscher FJ 3rd. Targeting histone deacetylase complexes via KRAB-zinc finger proteins: the PHD and bromodomains of KAP-1 form a cooperative unit that recruits a novel isoform of the Mi-2alpha subunit of NuRD. Genes Dev 2001;15:428-43

Schultz DC, Ayyanathan K, Negorev D, Maul GG, Rauscher FJ 3rd. SETDB1: a novel KAP-1-associated histone H3, lysine 9-specific methyltransferase that contributes to HP1-mediated silencing of euchromatic genes by KRAB zinc-finger proteins. Genes Dev 2002;16:919-32

Shuai K, Liu B. Regulation of gene-activation pathways by PIAS proteins in the immune system. Nat Rev Immunol 2005;5:593-605

Tan J, Hall SH, Hamil KG, Grossman G, Pwtrusz P, Liao J, Shuai K, French FS. Protein inhibitor of activated Stat-1 is a 
nuclear receptor coregulator expressed in human testis. Mol Endocrinol 2000;14:14-26

Tommerup $\mathrm{N}$, Vissing $\mathrm{H}$. Isolation and fine mapping of 16 novel human zinc finger-encoding cDNAs identify putative candidate genes for developmental and malignant disorders. Genomics 1995;27:259-64

Tussie-Luna MI, Bayarsaihan D, Seto E, Ruddle FH, Roy AL. Physical and functional interactions of histone deacetylase 3 with TFII-I family proteins and PIASx $\beta$. Proc Natl Acad Sci USA 2002;99:12807-12

Underhill C, Qutob MS, Yee SP, Torchia J. A novel nuclear receptor corepressor complex, N-CoR, contains components of the mammalian SWI/SNF complex and the corepressor KAP-1. J Biol Chem 2000;275:40463-70

Vissing H, Meyer WKH, Aagaard L, Tommerup N, Thiesen $\mathrm{HJ}$. Repression of transcriptional activity by heterologous KRAB domains present in zinc finger proteins. FEBS Lett 1995;369:153-7

Zheng L, Pan H, Li S, Flesken-Nikitin A, Chen PL, Boyer TG, Lee $W H$. Sequence-specific transcriptional corepressor function for BRCA1 through a novel zinc finger protein, ZBRK1. Mol Cell 2000;6:757-68 